\title{
Le long trajet vers le Nord : de la traduction de la littérature de jeunesse française en suédois
}

Carina Gossas et Charlotte Lindgren

Collège universitaire de Dalécarlie

La littérature de jeunesse ${ }^{\mathrm{I}}$ constitue tant en Suède qu'en France un secteur florissant. Selon l'Institut suédois des livres pour enfants (Svenska Barnboksinstitutet, dorénavant "l'institut SBI»), qui présente dans son bulletin annuel ${ }^{2}$ des statistiques très complètes sur les publications de littérature de jeunesse en Suède, le nombre de celles-ci ne cesse en effet d'augmenter et représente environ Io \% de la production totale des livres. Pour la France, ce taux atteint les I 7 \% selon le Centre National du livre (sans compter les bandes-dessinées et les mangas). En France, Poslaniec parle d'un nouvel "âge d'or » de la littérature de jeunesse depuis le milieu des années 2000 , avec de plus en plus de livres vendus, un chiffre d'affaire en hausse mais aussi un " un dynamisme et une créativité remarquables [...], un formidable vivier d'auteurs et illustrateurs jeunesse » (Poslaniec, 2008 : 94-95). Or — ce qui nous intéresse particulièrement dans le cadre de cet article — on sait que la littérature de jeunesse a toujours appartenu à un domaine hautement international depuis son apparition au XVIII ${ }^{\mathrm{e}}$ siècle ${ }^{3}$ : les livres ont été échangés entre les pays et les cultures grâce à des adaptations et des traductions.

Dans des études antérieures, nous avons pu constater que les échanges franco-suédois dans le domaine de la littérature de jeunesse contemporaine de qualité étaient plutôt unidirectionnels et que très peu de la production contemporaine en français était traduite en suédois (Gossas \& Lindgren, 20II ${ }^{4}$. Si quelques «grands classiques » de la littérature suédoise de jeunesse se trouvent sur le marché français depuis longtemps (ibid.), il n'en est pas de même pour certains "grands auteurs » en français sur le marché suédois : ni la comtesse de Ségur, ni Marcel Aymé, par exemple, n'ont trouvé une place dans ledit système

How to cite this book chapter:

Gossas, C. et Lindgren, C. 20 I 5 . Le long trajet vers le Nord : de la traduction de la littérature de jeunesse française en suédois. In: Cedergren, M. et Briens, S. (eds.) Médiations interculturelles entre la France et la Suède. Trajectoires et circulations de 1945 à nos jours. Pp. 234-247. Stockholm: Stockholm University Press. DOI: http://dx.doi.org/IO.I6993/ bad.r. License: CC-BY 
littéraire. La comtesse de Ségur (I799-I 874) est un incontournable auteur classique en France et ses livres sont constamment réédités. Pour un public suédois, par contre, la comtesse de Ségur est peu, voire pas connue, car il n’y a jamais eu, comme le montre Kåreland, de véritable traduction de ses œuvres en Suède (Kåreland, 2000 : 33-34). Il n’y a eu que quelques versions raccourcies publiées par de petites maisons d'édition sans grand retentissement dans les articles des critiques littéraires canonisants. Il en va de même pour Marcel Aymé (I902-I967). Des fameux Contes $d u$ chat perché, régulièrement réédités depuis 1934 pour un jeune public francophone, une seule traduction (Påfågeln och andra sagor) a été publiée en Suède en I954, de sorte qu'Aymé est aussi plus ou moins ignoré du public suédois. Enfin, pour prendre deux auteurs contemporains parmi les plus estimés, l'on voit que Marie-Aude Murail a publié en France une trentaine de romans, dont un seul est traduit en suédois (Simple / Pucko) et parmi environ vingt-cinq romans de Marie Desplechin, un seul existe en suédois (La belle Adèle / Ihop på låtsas). Il n'y a pas, à notre connaissance, d'étude approfondie sur la place de la littérature de jeunesse française à l'étranger, à laquelle comparer ces observations. De plus, contrairement à la situation scandinave, il n'y a pas vraiment de politique active en France pour promouvoir la littérature de jeunesse française à l'étranger. Ce que l'on sait, toutefois, est que « les Anglo-saxons trouvent [les livres français pour la jeunesse] 'trop sophistiqués', pas assez 'enfantins'» (Beau \& Lévêque, 2008 : 52), avis qui, selon nous, a longtemps été partagé en Suède. Toujours estil que dans les listes publiées par l'institut SBI, le français a toujours fait partie des langues sources, privilège partagé par simplement une dizaine de langues sources, comme nous le verrons ci-dessous. De plus, comme le constate le même institut dans son bulletin de 20 I3 $_{3}$, le français s'est démarqué cette année car les traductions de cette langue ont augmenté. Il semble donc légitime de parler d'une ouverture vis-à-vis des productions contemporaines en français, augmentation qui a lieu à un moment, on le verra, où le marché suédois pour la jeunesse est plus diversifié que jamais.

Ces observations nous ont conduit à mener cette enquête pilote sur la circulation de la littérature de jeunesse en français vers la Suède et sur la place qu'elle y occupe. Le but de l'article est d'abord de déterminer de façon quantitative la place des livres français traduits sur le marché suédois, pour ensuite détailler les types de livres qui se cachent derrière les chiffres, afin de voir si la littérature de jeunesse contemporaine française de qualité y trouve sa place. Si tel est le cas, nous voudrions tenter 
de caractériser les normes préliminaires concernant surtout les choix de titres traduits. Y-a-t-il une prédilection pour un support (livre illustré ou roman) ? Traduit-on tous les ouvrages d'un auteur ou est-ce fait de façon plus éparpillée ? Les traductions sont-elles mentionnées dans la presse ? Enfin, nous tenterons de mettre les traductions en relation avec les tendances actuelles dans le domaine de la production nationale suédoise.

\section{Le système littéraire suédois : un système ouvert à la traduction?}

Notre étude s'appuie sur la théorie des polysystèmes (voir par exemple Even-Zohar, I990) et sur celle de la traductologie descriptive (en anglais "DTS », Descriptive Translation Studies, voir Toury, I995 et 20I 2) qui partent du fait que la sphère culturelle au sens large contient différents systèmes dynamiques dans le temps et dans l'espace, et de l'idée que l'activité traduisante peut être décrite en termes de "normes » qui sont non pas des règles normatives mais plutôt des pratiques régulières. Les normes sont de différents types et dans cet article, nous nous intéressons aux normes dites préliminaires, qui concernent les types de textes traduits, leur origine, la langue source, etc. Dans le système littéraire, les livres ont une place centrale ou périphérique : la place au centre est prise par les canons de la littérature, et la place à la périphérie est prise par des livres qui ont un statut culturel moindre et que l'on appelle parfois populaires. Il apparaît que, souvent, la littérature traduite et la littérature de jeunesse ont une place semblable (périphérique) dans le système littéraire. L'idée que nous suivons est que les livres ne peuvent fructueusement être étudiés en dehors du système littéraire auquel ils appartiennent. Even-Zohar (I990: 50) a aussi montré que le système des œuvres traduites est souvent, malgré sa place périphérique, beaucoup plus actif que le système littéraire auquel il appartient, même s'il y a bien évidemment des différences selon les pays. Une idée centrale est que les systèmes sont en relation les uns avec les autres et qu'ils ont la possibilité de s'influencer : les livres traduits peuvent ainsi influencer les livres du système littéraire dans lequel ils arrivent. Il est donc intéressant de voir quels sont les livres qui sont traduits, quand et par qui.

À l'instar d'Even-Zohar (I990: II), nous soutenons que le système suédois est un système apparemment ouvert. Selon Even-Zohar, un système fermé est un système où les traductions ne jouent aucun rôle ou un rôle moindre (la production nationale étant autosuffisante) ; alors 
que dans un système ouvert la traduction a un rôle important à jouer et contribue à importer des modèles littéraires de l'extérieur. Étant donné que près de la moitié des livres de jeunesse publiés en Suède sont traduits, il semble être justifié de dire que le système est très ouvert ${ }^{5}$, même si, et il est important de le souligner, des données quantitatives ne disent a priori rien sur une éventuelle influence réelle. En même temps, on remarque vite que très peu de langues sont représentées. Plus que la moitié des livres traduits ont l'anglais comme langue source (entre 60 et $80 \%$ selon l'année), ce qui n'a peut-être rien d'étonnant en soi vu la dominance générale de la culture anglophone et le statut de l'anglais en Suède. Après l'anglais les autres langues scandinaves occupent une place centrale, sans doute à cause de la proximité culturelle. Au total, on ne compte qu'environ dix langues sources dans les statistiques de l'Institut SBI. La place du français sera discutée dans la section suivante où nous verrons que cette langue a toujours fait partie de cette petite famille qui a le privilège d'être traduite en suédois.

Nous avons consulté le catalogue numérique de la Bibliothèque Royale de Suède (Kungliga biblioteket, $K B$ ) en cherchant les livres en suédois classés comme littérature de jeunesse, de langue source française. Comme nous nous intéressons principalement à la période de 2000-20I3 nous avons recensé tous les livres pour cette période, tout en les classant en catégories très larges. Nous donnerons aussi ci-dessous un bref aperçu général d'une plus longue période (I970-2000), mais en étant dans l'impossibilité dans le cadre de cet article de présenter des données chiffrées, par manque de place.

Pour compléter les informations de la Bibliothèque Royale de Suède, nous avons consulté les statistiques de l'institut SBI. Ces statistiques sont publiées chaque année et montrent que la part des livres de jeunesse traduits en suédois depuis une autre langue est d'environ $50 \%$ des nouvelles publications, et cela de façon relativement constante dans le temps. La production nationale suédoise est toutefois relativement importante. Le tableau I présente le total des publications, le pourcentage de traductions et les traductions du français.

Comme nous l'avons déjà dit, on voit dans ce tableau une augmentation relativement constante du nombre de publications de jeunesse en suédois depuis l'an 2000. De plus, la part des livres traduits correspond bien à ce que nous avons avancé ci-dessus, c'est-à-dire que le système littéraire suédois paraît être, tout au moins d'un point de vue quantitatif, un système ouvert. L'on voit aussi que la part des livres traduits du français est plus ou moins la même, avec toutefois une augmentation 
en 2013 , pour passer à $6 \%$ - presque le double du pourcentage de l'an 2000.

\section{Les traductions du français vers le suédois : bref rappel historique et tendances actuelles (2000-2013)}

Ces quarante dernières années, les livres de jeunesse en français traduits en suédois ont principalement été constitués par six groupes de livres. Il existe tout d'abord une liste assez courte de livres considérés comme des classiques, qui ont été traduits et retraduits en suédois et constituent la base fondatrice de l'ensemble étudié dans cet article. Ce sont principalement les ouvrages les plus connus de Jules Verne et deux ouvrages d'Alexandre Dumas, considérés de nos jours comme de la littérature de jeunesse (Les trois mousquetaires / De tre musketörerna, Le comte de Monte-Cristo / Greven av Monte Cristo). Les bandes-dessinées franco-belges : Tintin, Spirou, les Schtroumpfs, Lucky Luke et Astérix, sont aussi très présentes dans les listes annuelles présentées par l'institut SBI.

Tableau 1. Le nombre de traductions et de traductions du français dans la publication annuelle de livres pour enfants en Suède (2000-20I3) selon l'institut SBI.

\begin{tabular}{|c|c|c|c|c|}
\hline & Total & Traductions & \multicolumn{2}{|c|}{ Dont des traductions du français } \\
\hline 2013 & I770 & $4 \mathrm{I} \%$ & 43 & $6 \%$ \\
\hline 2012 & $\mathrm{I} 76 \mathrm{I}$ & $46 \%$ & $5 \mathrm{I}$ & $5 \%$ \\
\hline $20 I I$ & I 747 & $46 \%$ & 39 & $4 \%$ \\
\hline 2010 & $\mathrm{I} 663$ & $50 \%$ & $2 \mathrm{I}$ & $2,5 \%$ \\
\hline 2009 & 1750 & $56 \%$ & 39 & $4 \%$ \\
\hline 2008 & I $82 \mathrm{I}$ & 6 I $\%$ & 37 & $3 \%$ \\
\hline 2007 & I 680 & $52 \%$ & 32 & $3,5 \%$ \\
\hline 2006 & I 5 I 5 & $58 \%$ & I9 & $2 \%$ \\
\hline 2005 & I493 & $55 \%$ & 37 & $4,5 \%$ \\
\hline 2004 & I 4 I 2 & $50 \%$ & I9 & $2,5 \%$ \\
\hline 2003 & I326 & $49 \%$ & 25 & $4 \%$ \\
\hline 2002 & I 206 & $52 \%$ & I 2 & $2 \%$ \\
\hline $200 \mathrm{I}$ & I I 82 & $57 \%$ & 25 & $4 \%$ \\
\hline 2000 & I I 76 & $65 \%$ & 23 & $3,5 \%$ \\
\hline
\end{tabular}


Les livres illustrés sont presqu'exclusivement représentés par Babar et Barbapapa. Quantitativement nombreux, on trouve des livres provenant des films de Disney (Cendrillon, La belle au bois dormant, etc.). Plus on remonte dans le temps, plus on trouve les contes de Perrault et les fables de la Fontaine. Enfin, deux romans dominent largement lesdites listes annuelles, ce sont le Petit Prince d'Antoine de Saint-Exupéry et la série des Six compagnons de Paul-Jacques Bonzon. Le Petit Prince est aussi présent dans des produits dérivés comme des imagiers ou des livres d'activités ludiques. Au contraire, si l'on se concentre maintenant sur les années 2000 , on voit que le genre des livres de jeunesse en suédois traduits du français a évolué dans les listes étudiées. À côté des tendances générales mentionnées ci-dessus et qui se maintiennent, on trouve des livres contemporains de qualité qu'on peut diviser en trois grands groupes: les livres pour tout-petits / albums / livres illustrés, sur lesquels nous insisterons particulièrement, les documentaires et les romans.

Avant de détailler les traductions, nous allons finalement présenter rapidement le système littéraire suédois actuel. Après 20I I - année charnière selon l'Institut SBI - la publication des livres illustrés en Suède est devenue de plus en plus riche et variée. De plus, le nombre total desdites publications est désormais plus grand que jamais. On trouve tout un éventail de livres, allant des produits de masse jusqu'à des produits de grande valeur artistique. Durant cette période, le marché du livre en général et celui des livres illustrés en particulier, semble donc ne pas cesser de croître en importance.

Si l'on se penche sur ces publications, on remarque l'établissement d'une nouvelle génération d'auteurs, principalement des femmes nées dans les années I970 ou I980. Leurs œuvres sont consacrées par des prix littéraires et par l'attention du public, à cause du renouvellement artistique et narratif qu'elles proposent (par exemple Emma Adbåge, Lisen Adbåge, Sara Lundberg ou Clara Persson). De plus, il y a plusieurs nouvelles maisons d'éditions, ce qui augmente la possibilité de traduire et publier. Deux tendances supplémentaires et complémentaires contribuent à élargir et à faire évoluer la forme du livre illustré au niveau national: d'un côté on voit paraître des livres s'adressant à un public plus diversifié (notamment des albums non spécifiquement pour enfants, domaine devant beaucoup à la traduction de quelques auteurs avant-gardistes norvégiens comme Gro Dahle, Svein Nyhus et Stian Holes). La parution en 2013 de Om detta talar man endast med kaniner d'Anna Höglund, prix de l'album de l'année 20 I 3 (le prix suédois Snöbollen) et nominé au prix August ${ }^{6}$, est un exemple très parlant qui montre que le 
livre illustré peut dorénavant s'adresser à un public plus âgé (adolescent voire adulte). D'un autre côté, il apparaît des albums sans texte ainsi que des livres à manipuler (livres tactiles et / ou sonores) ainsi que des livresjouets. Ici, les traductions jouent également un rôle important et il nous semble que le livre illustré suédois a plus ou moins rattrapé l'avance en matière de variété et de créativité, qu'avaient d'autres pays, par exemple la France (Van der Linden, 2006). Comme nous le verrons ci-dessous les albums et livres illustrés représentent une bonne partie des titres traduits du français. Les titres français sélectionnés qui arrivent sur le marché suédois correspondent donc, du moins en partie, aux tendances que nous venons de mentionner, le marché s'étant modernisé et les titres étant choisis, du moins en partie, en fonction du marché.

\section{Les livres pour tout-petits, les albums et les livres illustrés}

Pour la période 2000-20I3, ces livres (dorénavant regroupés sous le terme de "livres illustrés ${ }^{7}$ ) constituent un champ relativement dynamique dont l'évolution peut être favorable aux traductions. Du français sont traduits des albums d'éveil ou «interactifs » (tactiles et / ou sonores) pour les tout-petits ainsi que des albums très " artistiques " s'adressant à un public plus âgé. Certains titres sont présentés dans la presse et reçoivent une bonne critique. Voyons maintenant quelques auteurs et livres existant dorénavant en suédois, en commençant par les livres pour les tout-petits. Il y a des livres en carton, des livres à toucher, des livres- éveil etc. C'est un investissement économique sûr mais la qualité artistique est au final variable. Les éditeurs en appellent parfois aux résultats d'études scientifiques contemporaines sur les capacités cognitives des bébés et petits enfants pour vendre ce genre de livres. Il nous semble que la traduction a un rôle important dans ce groupe : les livres en français viennent effectivement combler un manque de production nationale en suédois. Nous ne mentionnerons pas ici plus spécifiquement les imagiers classiques, certes très nombreux, mais préférons attirer l'attention sur ce qu'il y a de plus novateur. La collection Mes petits imagiers sonores de Marion Billet ( $\mathrm{O}_{-3}$ ans) est, selon Van der Linden (20I I : 49) composée d'imagiers d'un genre nouveau, mélange de documentaires et imagiers, qui permettent d'associer un son à une image. La collection semble avoir du succès en Suède. On avait en effet commencé avec un des exemplaires les moins typiques, Fåglar / Les oiseaux, en 20I I (en même temps qu'on publiait des livres sonores pour les adultes pour reconnaître les chants des oiseaux) et Husdjur / Mes 
animaux. À l'heure actuelle la série Nyfikna öron chez Rabén et Sjögren comprend douze titres, dont trois figuraient parmi les 200 livres offerts à la princesse héritière de Suède Estelle à sa naissance, de la part du gouvernement suédois (livres sélectionnés par le directeur de l'Institut SBI Jan Hansson et une directrice d'école Lena Runesten ${ }^{8}$. Au même moment, dans la production nationale suédoise on peut noter l'apparition des livres qui permettent d'écouter de la musique (par exemple Svenska Barnvisor de Tina Ahlin, " un livre de karaoké pour les familles avec enfants " selon l'éditeur). Un genre de livre complètement inédit, traduit en 25 langues et couronné par de nombreux prix littéraires dans le monde est l'ouvrage d'Hervé Tullet Un livre (20I0). Tullet réinvente le rapport aux lecteurs et encourage l'activité de l'enfant, auquel il est demandé de tapoter sur les pages, applaudir, etc., durant la lecture. Il est paru en suédois en 20I I (sous le titre En bok)9.

Parmi les albums pour les petits, la série de Pomélo, de Ramona Badescu (texte) et Benjamin Chaud (illustrations) a aussi eu du succès. Des I 3 livres en français, il existe en suédois trois livres pour les $0-3$ ans (Pomelo den lilla elefanten 2003, Pomelo är kär 2004, Pomelo blir stor 20II) et deux pour les 3 à 6 ans (Pomelo och motsatsorden 20I I, Pomelo och färgerna 20I2). En 2009 le petit éléphant est même monté sur la scène du théâtre grâce à Sophia Segrell och Lisa Kjellgren (du théâtre Teater Tittut). Benjamin Chaud est aussi en première ligne en Suède pour plusieurs livres dont il est l'auteur Adieu Chausette 2010 / Min kanin Sockan 20II, Une chanson d'ours 20II / Björnens sång 20I2, Coquillages et petit ours 2012 / Lilla Björn på semester 2013. Il est également l'illustrateur de deux livres d'auteurs suédois, à savoir Snömannen d'Eva Susso (traduit en français par Jean-Baptiste Coursaud en 2013) et Tro på tomten de Lotta Olsson. Il a aussi illustré un roman Tusen tips till en fegis d'Eva Susso. Comme le remarque l'Institut SBI en 2013 , ce genre de collaboration internationale se fait de plus en plus souvent. Il est intéressant de voir que le paratexte des deux albums sur l'ours met nettement en avant l'origine française des ouvrages. Dans la plupart des cas le fait que ce soit un livre traduit du français n'est en effet pas mis en avant. Sur le quatrième de couverture de Björnens sång l'auteur est présenté comme "un des meilleurs illustrateurs français ». Sur celle de Lilla Björn på semester le lecteur est informé sur les louanges faites dans la presse suédoise régionale et nationale quant au précédent livre : "Bedårande godnattsaga!'irrésistible histoire à lire le soir au coucher' ", "Äkta bilderboksäventyr! 'vraie aventure de livre illustré’ ", "Årets bilderbokshöjdare ! 'le livre illustré de l'an- 
née' », "Mästerverk! ‘ un chef d'œuvre' », "Ögonfröjd! 'un plaisir des yeux’ ", "Överdådig! 'fastueux’ ", "Rolig elegant ! 'drôle et élégant' ", "Härlig och varmbjärtad! 'sympa et chaleureux' ». Remarquons, enfin, que les livres de Benjamin Chaud ont été presque immédiatement traduits en suédois et que ce n'est probablement que le début.

Une autre auteure dont plusieurs albums sont sortis récemment en suédois est Stéphanie Blake, qui a publié de nombreux livres pour les tout-petits, en particulier pour la collection Loulou et Cie à l'École de loisirs. Ce sont les albums de son lapin Simon, traitant avec humour, une simplicité graphique et des couleurs vives, divers thèmes et sentiments de la vie quotidienne, qui sont parus en Suède. Huit ouvrages ont été publiés par la maison Bergh (pour les $0-3$ ans ou pour les $3-5$ ans, selon le thème traité) : Caca Boudin 2002 / Bajskorven 2008, Poux 2009 / Löss 2009, Au loup 2005 / Hjälp en varg 2009, Donner c'est donner 2007 / Bytt är bytt 2010, Aaaah! pas le dentiste ! 2010 / Nej! Inte tandläkaren 20I1, Bébé cadum / Dumma lillebror 2012, Non pas le pot 20I I Inte pottan 2013, Non pas dodo! 2010 / Kan inte sova 20I4. Nej, inte tandläkaren est également publié chez En bok för alla ${ }^{\mathrm{I}}$. Il y a aussi eu, finalement, une adaptation suédoise de Caca boudin pour le théâtre en 2010 (par Hans Ung et Björn Johansson, au théâtre régional dans la région de Blekinge / Kronoberg). On voit que le premier livre est paru en suédois six ans après la version originale, et que, même si les traductions ne suivent pas l'ordre chronologique des parutions en français, une série s'est implantée. Le paratexte ne met pas en avant l'origine étrangère du livre et ne fournit pas de présentation de l'auteur. Son succès auprès du public suédois est signalé par exemple dans la présentation qu'Adlibris, une des plus grandes librairies en ligne suédoises, fait de Kan inte sova : " ny bok om kultkaninen från boken Bajskorv. 'Un nouveau livre sur le lapin culte du livre Caca Boudin' ». La maison Bergh a également fait traduire deux albums humoristiques du jeune auteur Michaël Escoffier, Bonjour docteur 2010 / Hos doktorn 20 Io et Un mammouth dans le frigo $20 \mathrm{I} \mathrm{I} \mathrm{/} \mathrm{En} \mathrm{mammut} i$ kylskåpet 20I I. Le jour où j'ai perdu mes superpouvoirs 2013 / Dagen då jag förlorade mina superkrafter du même auteur vient de paraître chez Opal.

Nous voulons enfin mentionner quelques livres illustrés qui s'adressent à un public plus âgé ou même adulte. Il s'agit de livres avec des illustrations soit poétiques, soit réalistes. On remarque que ces livres sont souvent parus dans de petites maisons d'éditions. Le livre très poétique sur la solitude, la vieillesse et la mort, La petite vieille du vendredi (20I2) de 
Marie Moinard, elle-même directrice de la maison d'édition Des ronds dans l'O, a été traduit en 20I 3 sous le titre de Den gamla damen som alltid kom på fredagen chez Epix, petite maison d'éditions spécialisée en bandes-dessinées pour adultes. Le livre est recommandé à partir de 5 ans. Pour la même tranche d'âge (de 3 à 6 ans dans le catalogue suédois, à partir de 5 ans dans le catalogue français) a paru Reine 2008 / Drottningen de Jacqueline Delaunay chez Turbine / Massolit Förlag.

Il existe dans la production contemporaine en français beaucoup de réécritures humoristiques des contes qui permettent de prendre de la distance. Un livre de cette catégorie a été publié en suédois en 2013 , à savoir Madame le Lapin blanc / Den vita kaninens fru (pour les 6-9 ans, $\mathrm{ABC}$ förlag), hommage à et réappropriation d'Alice au pays des merveilles. L'humour et les illustrations minutieusement détaillées lui ont valu le prix Pépite de l'album du Salon du livre et de la presse jeunesse de Montreuil en 20I2. Le côté artistique est également prépondérant dans William Morris bok de Benjamin Lacombe (texte) et Agata Kawa (illustrations), Alvina förlag. Selon une critique du quotidien suédois Svenska Dagbladet (SvD), même si ce livre est destiné surtout à un lectorat adulte, avec un bagage culturel certain, les illustrations dégagent un tel chatoiement que n'importe quel humain intéressé par les images sera fasciné [notre reformulation] ${ }^{\mathrm{II}}$.

\section{Les documentaires}

Les livres documentaires ne font pas partie du système littéraire suédois pour la jeunesse depuis très longtemps, et surtout pas concernant les petits enfants. Comme Marion Billet mentionnée à propos des livres pour tout-petits, beaucoup de documentaires traduits du français sont présents dans les listes annuelles publiées par SBI. Un exemple est la série destinée aux enfants curieux (série appelée Nyfiken chez Bonnier Carlsen), qui est destinée à un public double, puisque les enfants peuvent soit écouter un lecteur la lire à voix haute, soit regarder et commenter les images comme dans un imagier (voir par exemple Les dinosaures / Nyfiken på dinosaurier de Pascale Hédelin (texte) et Didier Balicevic (illustrations). Dans ce domaine, l'on remarque aussi la longue tradition française de produire des encyclopédies. Pour des enfants un peu plus âgés, Le livre des pourquoi (2013) de Martine Laffon et Hortense de Chabaneix a été traduit en 2013 par Ordalaget Bokförlag (Stora boken om varför) et Mon premier Larousse des comment (2004) a été traduit en 20I I chez Rabén \& Sjögren (Stora frågeboken). 


\section{Les romans}

Les romans traduits du français sont un peu plus rares. Il existe beaucoup d'auteurs nationaux très appréciés et reconnus, et les traductions des grandes séries anglophones ne laissent pas non plus une grande place aux œuvres venues d'autres cultures. On trouve dans les listes de l'Institut SBI des livres pour les enfants qui viennent d'apprendre à lire ou apprennent à lire (environ 6 à 9 ans) : un bon exemple est Quel stress pour la maîtresse ! / Frökens nya klass de Jo Hoestlandt (2010). Ce livre adopte la perspective de la maîtresse d'école un jour de rentrée scolaire, alors que d'habitude on trouve le point de vue inverse (celui des enfants). Les illustrations d'Eric Gasté ont un style humoristique. En France, Jo Hoestlandt est une auteure très productive. Un autre exemple sont les livres d'Hubert Ben Kemoun, lui aussi un auteur très productif dont les ouvrages sont illustrés de façon humoristique (par exemple Nico, tous les jours c'est foot! / Fotbollsstjärnan).

Pour des lecteurs un peu plus expérimentés (9 à I 2 ans) on peut prendre l'exemple des deux livres d'Anne Plichota (Oksa Pollock, l'inespérée (tome I) / Det sista hoppet et Oksa Pollock, la forêt des égarés (tome 2) / De vilsnas skog). Ils sont sortis en Suède en 20I2. En France, plusieurs tomes sont publiés après les deux premiers mais ils ne sont pas encore traduits. Il est ici intéressant de remarquer que la critique dans le quotidien suédois précité a été plutôt glaciale (le critique parle d'ouvrage "mauvais » et d' "échec ${ }^{\text {I2 }}$ ) alors qu'un critique en France parlait d'" une écriture limpide mais soignée qui donne à cette saga addictive de sérieux atouts pour s'imposer comme un classique ${ }^{\mathrm{I}}{ }^{3}$. Citons aussi l'auteur Ludovic Huart, qui en France a sorti des livres aux titres effrayants (L'abominable carnaval du grand théâtre des monstres, La monstrueuse histoire d'un petit garçon moche et d'une petite fille vraiment très laide, La funeste nuit d'un loup en peluche qui ne devait plus murmurer à l'oreille des enfants) et dont L'épouvantable Noël du vieux monsieur barbu tout de rouge vêtu. Ou le jour où le Père Noël cessa de croire aux enfants sages et obéissants est traduit en suédois (Den förfärliga julen: den kusliga berättelsen om den skäggige gamle rödklädde herrn eller dagen då jultomten slutade tro på snälla och lydiga barn).

Pour les I 2 à I 5 ans, on trouve un roman écrit par une des auteurs de jeunesse les plus connues en France, Marie-Aude Murail, et mentionnée ci-dessus. La perspective donnée est celle d'un jeune adulte handicapé mental. Aucun autre de ses livres n'a été traduit en suédois. Mentionnons également Marie Desplechin, auteure aussi connue pour 
ses romans pour adultes, avec La belle Adèle / Ihop på låtsas. On remarque que ce livre s'adresse en français à un public un peu plus jeune, avec une couverture illustrée, alors que le livre en suédois s'adresse à un public un peu plus âgé, avec une couverture qui fait plus « jeune adulte».

\section{Conclusion}

Cette étude avait pour but de montrer quelles sont les normes préliminaires concernant les choix des livres de jeunesse français traduits en suédois. Nous avons vu que l'intérêt qu'ont porté ces dernières années les passeurs des livres suédois à la production contemporaine en français s'est surtout concentré sur les livres illustrés au sens large. D'autres types d'œuvres sont représentés dans les listes de traductions (les documentaires et les romans), mais de façon moins flagrante. Nous avons aussi montré que, comme c'est le cas lorsque les ouvrages suédois sont traduits en français, les éditeurs privilégient l'établissement de séries. Toutefois, dans les listes établies par l'Institut SBI, ce fait est plus rare dans le sens inverse étudié ici. Ne faisons pas l'erreur de simplement regarder les chiffres, car, même si les livres en français traduits ne sont pas extrêmement nombreux, ils ont le mérite d'exister, ce qui n'était pas le cas avant, comme nous l'avons vu (du moins, il ne s'agissait pas des mêmes genres de livres mais plutôt de bandes dessinées et de classiques, au sens large du terme, comme les ouvrages de Jules Verne, Saint-Exupéry, Disney, etc.). De plus, on est passé d'une période pendant laquelle leur traduction en suédois n'était pas donnée a priori à une période où elle est remarquée, recommandée, et parfois même consacrée par les critiques littéraires et les professionnels du livre. Depuis le début des années 2000, il semble donc y avoir eu un intérêt continu pour les livres illustrés et albums contemporains de qualité venus du français. Il serait maintenant intéressant de compléter l'étude que nous venons de présenter en examinant, dans une prochaine étape, les stratégies de traduction des livres de jeunesse français en suédois au niveau textuel et iconique.

\section{Notes}

I. Le propos de cet article n'est pas de discuter la définition de la littérature de jeunesse. De plus, dans les ouvrages, articles, colloques ou discussions sur la question, une telle définition est souvent remise en cause ou qualifiée de difficile, de fluctuante. C'est pourquoi, en utilisant le terme de " littérature de 
jeunesse ", nous renvoyons aux livres écrits et édités pour les enfants et lus par les enfants (voir la discussion de Nières-Chevrel 2009 : I 5).

2. En suédois Bokprovning. (http://www.sbi.kb.se/en/Resources/Trends-andstatistics/Book-tasting/)

3. Voir par exemple Nières-Chevrel (2009:32-54) pour un aperçu historique.

4. Concernant la traduction du suédois vers le français, voir aussi par exemple Gossas et Lindgren (20I4), Lindgren (20I2), Lindgren et al. (2007) et Renaud et al. (2007).

5. D'autres auteurs ont émis l'hypothèse que la traduction se fait principalement dans le sens des langues très parlées vers les langues plus rares (appelées par Casanova "dominantes " et "dominées ", 2002 : 9). On attendrait alors un nombre plus important de traductions du français au suédois et non le contraire, comme cela a été le cas dans le domaine qui nous concerne.

6. Le prix August est un prix littéraire suédois prestigieux en souvenir de l'écrivain August Strindberg.

7. «L'album se différencie du livre illustré par l'utilisation qui est faite de l'image » (Bibliothèque Nationale de France, http://classes.bnf.fr/rendezvous/pdf/ albums.pdf).

8. http://www.regeringen.se/content/I/c6/19/33/92/eo5cb29d.pdf

9. En 20I4 a paru un deuxième titre du même auteur Couleurs / Färger.

Io. Cette association 'Un livre pour tous' veut depuis I976 (jusqu'en 2007 avec des subventions gouvernementales) rendre accessibles à tous des livres de qualité, dans le but de promouvoir la lecture grâce à des publications bon marché.

I I. http://www.svd.se/kultur/litteratur/en-frojd-for-skonhetstorstande-sjalar_ $6685872 . s v d$

I 2. http://www.svd.se/kultur/litteratur/ny-fantasy-idiotforklarar-lasaren_.svd

I3. http://www.lefigaro.fr/livres/20I 2/I I/06/03005-20 I 2 I I06ARTFIGo0426oksa-pollock-en-poche.php

\section{Bibliographie}

Beau, N. \& Lévêque, M. (2008), "Les livres français pour la jeunesse traduits à l'étranger : esquisses d'une enquête ", in Diament, N., Gibello, C. Kiéfé, L. (éd.), Traduire les livres pour la jeunesse : enjeux et spécificités. Paris : Hachette, BnF/CNLPJ - la Joie par les livres : 43-54.

Casanova, P. (2002), "Consécration et accumulation de capital littéraire [La traduction comme échange inégal] », Actes de la recherche en sciences so- 
ciales I 44, septembre 2002, Traductions: les échanges littéraires internationaux : 7-20.

Even-Zohar, I. (I990), "The Position of Translated Literature within the Literary Polysystem ", Poetics Today I I :I :45-5 I.

Gossas, C. \& Lindgren, C. (2OI4), «Traduction et interculturalité : un exemple de livres illustrés suédois contemporains », in Reuter, H. (dir.), Le nord en français, Collection "Langage et société » du Centre international de phonétique appliquée de Mons (CIPA) : 39-52.

- (20II), "Svensk barnboksexport till Frankrike - trender och anpassning 1989-2009 ", Konferensvolym, IASS 20I0, 28:e studiekonferensen i International Association of Scandinavian Studies (IASS).

Kåreland, L. (2000), "Okänd i Sverige - älskad i sitt hemland. 200-års minnet av la comtesse de Ségurs födelse firas i Frankrike ». Barnboken 2000:I : 28-35.

Lindgren, C. (2012), "He speaks as children speak: more orality in translations of modern Swedish children's books into French? », in Fischer, M., Wirf Naro, M. (éd.) Translating Fictional Dialogue for Children. Berlin, Frank \& Timme Verlag für wissenschaftliche Literatur : I65-I 85 .

Lindgren, C., Andersson, C. \& Renaud, C. (2007), "La traduction des livres pour enfants suédois en français: choix et transformation ». Revue des livres pour enfants 234:87-93.

Nières-Chevrel, I. (2009), Introduction à la littérature de jeunesse. Paris : Didier Jeunesse.

Poslaniec, C. (2008), Des livres d'enfants à la littérature de jeunesse. Collection Découvertes Gallimard, Littératures : no 534 : Paris : Gallimard-BNF.

Renaud, C. Andersson, C. \& Lindgren, C. (2007), « L'image dans la traduction de livres pour enfants: défi ou soutien. Réflexions à partir de la traduction en français de quelques livres pour enfants suédois ". Revue des livres pour enfants $234: 94-$ IOI.

Toury, G. (I995), Descriptives Translation Studies and Beyond. Amsterdam: Benjamin.

- (2012), Descriptive Translation Studies - and Beyond. Revised edition. Amsterdam : Benjamin.

Van der Linden, S. (2006), Lire l'album. Paris : Atelier du poisson soluble.

- (20I I), Je cherche un livre pour un enfant - Le guide des livres pour enfants de la naissance à sept ans. Paris : coédition Gallimard Jeunesse-Éditions De Facto. 